\title{
Achieving stress-constrained topological design via length scale control
}

DOI:

10.1007/s00158-018-2019-y

\section{Document Version}

Accepted author manuscript

Link to publication record in Manchester Research Explorer

\section{Citation for published version (APA):}

Amir, O., \& Lazarov, B. (2018). Achieving stress-constrained topological design via length scale control. Structural and Multidisciplinary Optimization. https://doi.org/10.1007/s00158-018-2019-y

\section{Published in:}

Structural and Multidisciplinary Optimization

\section{Citing this paper}

Please note that where the full-text provided on Manchester Research Explorer is the Author Accepted Manuscript or Proof version this may differ from the final Published version. If citing, it is advised that you check and use the publisher's definitive version.

\section{General rights}

Copyright and moral rights for the publications made accessible in the Research Explorer are retained by the authors and/or other copyright owners and it is a condition of accessing publications that users recognise and abide by the legal requirements associated with these rights.

\section{Takedown policy}

If you believe that this document breaches copyright please refer to the University of Manchester's Takedown Procedures [http://man.ac.uk/04Y6Bo] or contact uml.scholarlycommunications@manchester.ac.uk providing relevant details, so we can investigate your claim.

\section{OPEN ACCESS}




\title{
Achieving stress-constrained topological design via length scale control
}

\author{
Oded Amir · Boyan S. Lazarov
}

Received: date / Accepted: date

\begin{abstract}
A new suite of computational procedures for stressconstrained continuum topology optimization is presented. In contrast to common approaches for imposing stress constraints, herein it is proposed to limit the maximum stress by controlling the length scale of the optimized design. Several procedures are formulated based on the treatment of the filter radius as a design variable. This enables to automatically manipulate the minimum length scale such that stresses are constrained to the allowable value, while the optimization is driven to minimizing compliance under a volume constraint - without any direct constraints on stresses. Numerical experiments are presented that incorporate the following : 1) Global control over the filter radius that leads to a uniform minimum length scale throughout the design; 2) Spatial variation of the filter radius that leads to local manipulation of the minimum length according to stress concentrations; and 3) Combinations of the two above. The optimized designs provide high-quality trade-offs between compliance, stress and volume. From a computational perspective, the proposed procedures are efficient and simple to implement: essentially, stress-constrained topology optimiza-
\end{abstract}

The first author received financial support from the Israeli Science Foundation, grant number 750/15. The work of the second author was partially performed under the auspices of the U.S. Department of Energy by Lawrence Livermore National Laboratory under Contract DEAC52-07NA27344.

\section{O. Amir}

Faculty of Civil and Environmental Engineering, Technion - Israel Institute of Technology

Tel.: +972-4-8293041

E-mail: odedamir@technion.ac.il

\section{B. S. Lazarov}

Lawrence Livermore National Laboratory, Livermore, CA, US

School of Mechanical, Aerospace and Civil Engineering, The University of Manchester, UK

E-mail: bslazarov@gmail.com tion is posed as a minimum compliance problem with additional treatment of the length scale.

Keywords Topology Optimization · Stress Constraints · Length Scale

\section{Introduction}

Topology optimization of continuum structures has evolved into a very successful computational approach for optimizing the distribution of materials in a certain design domain. It is particularly useful for early, conceptual design stages when the aim is find structrual layouts that posess a good trade-off of weight, stiffness and strength. For extensive reviews of methods and procedures the readers are referred to the monograph by Bendsøe and Sigmund (2003) and to review articles (Eschenauer and Olhoff 2001, Sigmund and Maute 2013; Deaton and Grandhi 2014). Despite the maturity of topology optimization, the inclusion of stress constraints still stands out as a relatively challenging topic that attracts considerable research efforts. In this paper, we wish to shed light on the relation between peak stresses and the characteristic length scale of the optimized design. Subsequently, we suggest a suite of computational procedures that aim at finding the stiffest structural layout for a given volume, while constraining the level of the maximum stress by controlling the length scale of the design. Throughout the article we follow the density-based approach where the topology is defined by density variables attached to each discretization point (finite element in the current context) in the design domain. Nevertheless, the concepts hold also for other means of describing the topological layout, such as level-sets.

The main difficulty in imposing stress constraints is their local nature - the number of constraints is comparable to 
the number of design variables - both related to the resolution of the underlying finite element mesh. So far, the dominating strategy for removing this obstacle has been constraint aggregation, meaning that the large set of local stress constraints are aggregated into a small number of approximate, global constraints. Originally, Yang and Chen (1996) examined the use of both Kreisselmeier-Steinhauser (KS) functions and $p$-norm functions (Park 1995). They minimized either the global stress or a weighted sum of global stress and compliance, subject to a volume constraint. Duysinx and Sigmund (1998) suggested two global stress functions, the $p$-mean and the $p$-norm, and showed that the maximum local stress is bounded from above by the $p$-norm and from below by the $p$-mean. Later, a "block aggregation" of the stresses was suggested (París et al 2007, 2010). Subsequently, Le et al (2010) proposed regional stress measures where local stress constraints are grouped in interlacing regions according to their stress level. Using also a normalization with respect to the actual maximum stress, promising results were obtained, in particular for the benchmark case of the L-bracket: a layout that avoids the re-entrant corner by creating a smooth boundary with low curvature was generated. Several additional positive results based on aggregated or approximate stress measures have been published over the last few years, presenting various appealing extensions, improvements and refinements to this approach, for example (Holmberg et al 2013, De Leon et al 2015; Kiyono et al 2016, Verbart et al 2017; Lian et al 2017; Thore et al 2017; Sharma and Maute 2017, Zhang et al 2017, Oest and Lund 2017, Picelli et al 2018).

A more accurate yet also more costly approach is to consider an active set of local stress constraints, suggested by Duysinx and Bendsøe (1998) and later by (Bruggi and Venini 2008, Bruggi and Duysinx 2012). Interestingly, the design of the L-bracket with mixed FEM and local constraints (Bruggi and Venini 2008) differs from the typical result with a $p$-norm approximation. The deviation from the boundary near the re-entrant corner is smaller and hence the overall stiffness is higher. Local constraints were considered also by Pereira et al (2004) and by Fancello (2006) who solved the optimization problem using an augmented Lagrangian approach. In the context of level-set topology optimization, Allaire and Jouve (2008) minimized an integral measure of a power-law penalty of stress while Amstutz and Novotny (2010) replaced the large number of stress constraints with an external penalty functional, both achieving layouts that avoid stress concentrations at re-entrant corners. Other approaches for achieving stress-constrained designs are based on mimicking the damage that occurs in overstressed material (Verbart et al 2016); and on considering the actual damage or plasticity that evolve when the yield stress is exceeded (James and Waisman 2014; Amir 2017; Zelickman and Amir|2018).
The current contribution offers a different view on stress constraints. In traditional structural design, a candidate design is examined for stresses and if the maximum stress exceeds the allowable value then two remedies are common: 1) Sizing modifications that increase the cross-section of a certain member or branch; 2) Shape modifications that increase the radius of curvature at the vicinity of sharp corners. In the context of topology optimization, these two actions can be seen as a manipulation of the design's characteristic minimum length scale - either of its solid phase (namely, thickening of a member) or of its void phase (namely, increasing the radius of curvature on the boundary). Hence we wish to examine the influence of the imposed length scale, which is typically an input parameter to a topology optimization procedure, on the maximum stress of the optimized design. Based on this investigation, we formulate several computational procedures for automatically manipulating the minimum length scale such that stresses are constrained to the allowable value, while the optimization is driven to minimizing compliance under a volume constraint - without any direct constraints on stresses.

Before introducing more details about the proposed algorithms, it is necessary to provide a definition of the length scale in topology optimization. The definition utilized herein is based on the theory presented in Wang et al (2011), which states that the sub-domains of an optimized design that are occupied with solid and void phases with strictly enforced length scale, are defined as unions of disks in 2-D and balls in 3-D with a finite radius. The radius represents the imposed length scale and can be different for the two phases. An important conclusion arising from previous studies on stressbased topology optimization (De Leon et al 2015) and on optimization with physical processes that locally affect material properties (Madsen et al 2016), is that they do not impose global length scale on the optimized design. The effect of the additional restrictions is observed only locally around points and regions with active constraints, i.e., points with stress concentration and critically loaded structural members. On the other hand, the set of black-and-white designs, with length scale larger than the one obtained with local constraints, can be seen as a set of design envelopes satisfying the required bounds on the stress with the additional requirement for manufacturability.

In this paper, we propose procedures to control the length scale directly. Consequently, we impose either uniform length scale or local length scale, that adequately bound the maximum stresses, without constraining them directly. The potential advantages of the proposed approach are as follows: 1) The stress-constrained optimization follows the so-called 'robust' formulation that results in black-and-white designs with enforced length scale; 2) The enforcement of a length scale provides a theoretically justified strict upper bound on the stress; 3 ) The procedures utilize the simple optimality 
criteria algorithm and do not require a more complex optimizer; and 4) The procedures do not require to solve an adjoint state so the cost per design iteration is lower than for existing approaches, in particular in large-scale when iterative solvers are necessary. We show that the obtained layouts exhibit strength-to-weight trade-offs that are competitive in comparison to existing approaches.

The remainder of the article is organized as follows. In Section 2 we investigate the influence of the minimum length scale on the value of the peak stresses. The relation between the commonly used compliance functional and the stress functional is presented in Section 3 Computational procedures with adaptive length scale and spatially varying length scale are described and demonstrated in Sections 4 and 5 respectively. Section 6 is dedicated to a direct comparison to a state-of-the-art implementation of the popular $p$-norm approach. Finally, a discussion of the results and concluding remarks are given in Section 7

\section{The influence of length scale on the peak stresses}

The current study has two main purposes: first, to demonstrate the important effect of length scale on the peak stresses that appear in the response of an optimized design; and second, to derive numerical procedures that enable to constrain the peak stresses by directly controlling the length scale. In this section, we expose the significance of length scale via numerical examples based on classical test cases.

Throughout the article, we consider the very common minimum compliance topology optimization problem formulation. We follow the density-based approach (Bendsøe 1989, Bendsøe and Sigmund 2003) meaning that the topology is defined according to density values at discrete points in the design domain. Consistent length scale control is a necessary ingredient in the proposed procedures. In densitybased approaches, it is typically achieved by a sequence of operations that transform the freely distributed design field $\rho$ into a certain physical material distribution on which minimum length scale is guaranteed.

In the first step, the design is filtered by the well-known density filter (Bruns and Tortorelli 2001; Bourdin 2001). The result is the filtered density field $\widetilde{\rho}$,

$\widetilde{\rho}_{i}=\frac{\sum_{j \in N_{i}} w\left(\mathbf{x}_{j}\right) v_{j} \rho_{j}}{\sum_{j \in N_{i}} w\left(\mathbf{x}_{j}\right) v_{j}}$

for which we use a linear weighting function

$w\left(\mathbf{x}_{j}\right)=r_{\min }-\left\|\mathbf{x}_{j}-\mathbf{x}_{i}\right\|$

where $r_{\text {min }}$ is the specified filter radius; $v_{j}$ is the volume of the element $j ; \mathbf{x}_{i}$ is the position of the centroid of element $i$ and $\mathbf{x}_{j}$ is the position of the centroid of element $j$ which is in the neighborhood $N_{i}$ of element $i$. Thus it is assigned a positive weight $w\left(\mathbf{x}_{j}\right)$.

In the second step, smooth Heaviside projection functions are applied. These encourage a distinct 0-1 (or voidsolid) layout (Guest et al 2004; Xu et al 2010). Furthermore, by applying the robust topology optimization approach where eroded and dilated layouts are added to form a worst-case formulation, minimum length scale is guaranteed (Wang et al 2011). It was shown that for problems involving only stiffness and volume one can simply use the eroded design for quantifying the worst case for stiffness whereas the dilated design quantifies the worst case for volume (Lazarov et al 2016). The filtered density field is therefore projected to eroded and dilated densities respectively,

$\bar{\rho}_{i}^{\text {ero }}=\frac{\tanh \left(\beta_{H} \eta_{\text {ero }}\right)+\tanh \left(\beta_{H}\left(\widetilde{\rho}_{i}-\eta_{\text {ero }}\right)\right)}{\tanh \left(\beta_{H} \eta_{\text {ero }}\right)+\tanh \left(\beta_{H}\left(1-\eta_{\text {ero }}\right)\right)}$
$\bar{\rho}_{i}^{\text {dil }}=\frac{\tanh \left(\beta_{H} \eta_{\text {dil }}\right)+\tanh \left(\beta_{H}\left(\widetilde{\rho}_{i}-\eta_{\text {dil }}\right)\right)}{\tanh \left(\beta_{H} \eta_{\text {dil }}\right)+\tanh \left(\beta_{H}\left(1-\eta_{\text {dil }}\right)\right)}$

where $\beta_{H}$ governs the curvature of the smooth projection; $\eta_{\text {ero }}$ is the projection threshold for the eroded layout (e.g. $\eta_{\text {ero }}=0.6$ ); and $\eta_{\text {dil }}$ is the projection threshold for the dilated layout (e.g. $\eta_{d i l}=0.4$ ). The actual design that is intended for manufacturing is determined by an intermediate projection, with $\eta_{\text {int }}=0.5$. We note that when stiffness and volume are used as the counteracting objective and constraint, the latter does not need to be evaluated during the optimization because it does not represent the worst case for neither of them.

Based on a standard finite element discretization, the discrete form of the optimization problem is defined as

$$
\begin{aligned}
\min _{\rho} \phi & =\mathbf{f}^{T} \mathbf{u} \\
\text { s.t.: } & g=\frac{\sum_{e=1}^{N_{E}} \bar{\rho}_{e}^{d i l} v_{e}}{\sum_{e=1}^{N_{E}} v_{e}}-V_{d i l}^{\star} \leq 0 \\
& 0 \leq \rho_{e} \leq 1, \quad e=1, \ldots, N_{E}
\end{aligned}
$$

with: $\mathbf{K}_{\text {ero }} \mathbf{u}=\mathbf{f}$

where $\rho$ is the vector of density design variables that govern the material distribution; $\mathbf{f}$ is the vector of external forces; $\mathbf{u}$ is the vector of displacements; $N_{E}$ is the number of continuum finite elements in the design domain; $v_{e}$ is the volume of the $e$-th finite element; $V_{d i l}^{\star}$ is the available volume fraction for the dilated distribution; and $\mathbf{K}_{\text {ero }}$ is the stiffness matrix corresponding to the eroded material distribution. For evaluating the stiffness matrix $\mathbf{K}_{\text {ero }}$, Young's modulus of each element in the eroded layout is determined by the Modified SIMP interpolation rule (Sigmund and Torquato 1997),

$E\left(\bar{\rho}_{i}^{\text {ero }}\right)=E_{\text {min }}+\left(E_{\text {max }}-E_{\text {min }}\right)\left(\bar{\rho}_{i}^{\text {ero }}\right)^{p}$

where $E_{\min }$ is a relatively small positive number in order to avoid singularity of the stiffness matrix; $E_{\max }$ is Young's 
modulus of the material to be distributed; and $p$ is the SIMP penalization factor. For all numerical demonstrations in this article, $p=3$ was used. When using separate projections for stiffness and volume as we do in the current study, penalization in the SIMP manner is not completely necessary. This is because the two projections impose penalty on the stiffness and reverse penalty (high cost) on volume in the density range between $\eta_{d i l}$ and $\eta_{\text {ero }}$. However, adding penalty in the SIMP interpolation speeds up the convergence towards a distinct topological layout hence the overall number of design cycles is reasonable also for practical applications.

Based on the discussion in Wang et al (2011), it is clear that the minimum length scale obtained in the optimized design depends on two parameters - the filter radius $r_{\text {min }}$ and the threshold value $\eta$ that controls the projection. In principle, the values of $\eta_{\text {ero }}$ and $\eta_{\text {dil }}$ can be chosen to be asymmetrical w.r.t. the intermediate value $\eta_{\text {int }}=0.5$. Then the minimum length scale imposed on solid and void features will be different. However, this case was not examined thoroughly in the current study and the discussion is based on symmetric projections only.

Two test cases are utilized in order to demonstrate the effect length scale has on the value of peak stresses. The first is the classical L-bracket which is frequently used to evaluate stress-constrained topology optimization procedures. The design domain is based on a $200 \times 200$ grid of square finite elements with side length of 1.0, in which the top-right square of $120 \times 120$ elements is fixed to be void. The design domain is further padded with a layer of 9 void elements so that minimum length scale is controlled consistently also on the free boundaries of the design domain, as discussed by Poulsen (2003), Lazarov et al (2016) and Clausen and Andreassen (2017). The size of the extended domain was determined such that also large values of $r_{\min }$ can be accommodated. In order to avoid stress concentrations at the loading point, we distribute the load over 9 adjacent nodes at the corner, and pad the design domain locally with 3 layers of solid elements. This ensures that the load is connected to material also after the Heaviside projections. For large filter radii, the padded solid region is extended to 5 layers. The problem setup is presented in Figure 1.

In all optimization runs, a fixed number of 150 design iterations is used. The initial value of $\beta_{H}$ is 1.0 and it is multiplied by 1.25 every 10 iterations, up to a maximum value of 16 . The desired volume fraction of the intermediate design is $V^{\star}=35 \%$ of the volume of the L-shape only. The volume constraint is adapted every 10 iterations as follows: first the ratio between the desired volume $V^{\star}$ (to be imposed on $\bar{\rho}^{\text {int }}$ ) and the volume computed with $\bar{\rho}^{\text {int }}$ is found; then, the volume limit for the dilated design $V_{d i l}^{\star}$ is multiplied by the square root of this ratio. This drives the volume of the intermediate density towards the desired value $V^{\star}$, while controlling the dilated density by an actual con-

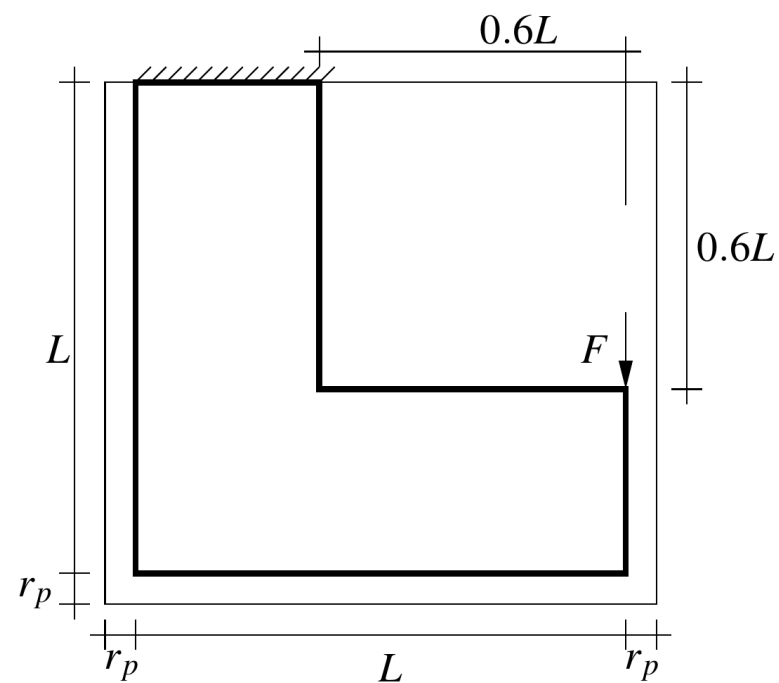

Fig. 1 Design domain and boundary conditions of an L-bracket. For consistent length scale on the boundary of the domain, the $L \times L$ square is padded with a void layer whose thickness is $r_{p}$.

straint. Throughout this section, the reported value of the maximum von Mises stress is computed as the true maximum on the intermediate design, meaning $\bar{\rho}^{\text {int }}$ replaces $\bar{\rho}^{\text {ero }}$ in Eq. 6. The final value of $\beta_{H}=16$ and $p=3$ are used. Finally, all optimization problems are solved with a classical Optimality Criteria approach, as implemented in popular codes for density-based topology optimization (Sigmund 2001; Andreassen et al 2011).

\subsection{The effect of filter radius}

The results of minimum compliance optimization of the Lbracket, with various filter radii and projection thresholds $\eta_{\text {dil }}=0.4$ and $\eta_{\text {ero }}=0.6$ are presented in Table 1 The actual minimum length scale imposed on solid and void features is presented alongside each optimized design using a circle whose diameter represents the length scale. It can be seen clearly that increasing the length scale equally for both solid and void phases by increasing the filter radius, has a dramatic effect on peak stresses. When varying the filter size from $r_{\text {min }}=3$ up to $r_{\text {min }}=17$, the optimized compliance increases monotonically w.r.t. the filter size. At the same time, the peak stress decreases monotonically as long as $r_{\text {min }} \leq 13$, then it begins increasing - presumably because of the significantly larger displacements that lead to larger strains. From a visual perspective, the increase in length scale is reflected in the number of bars and holes, in their minimum thicknesses, and in the curvatures of the boundaries. A small filter radius (e.g. $r_{\text {min }}=3$ ) leads to a layout consisting of thinner bars and infinite curvature in the re-entrant corner - leading to the best performance in terms of compliance. A larger filter radius (e.g. $r_{\min }=9$ ) leads to a layout with 
fewer, thicker bars and smaller curvatures in both external and internal boundaries - leading to better performance in terms of stresses.

The same trends can be observed also when asymmetric projection thresholds are utilized. These imply that the imposed length scale is different for solid and void. As mentioned above, the asymmetric case is not investigated thoroughly in this contribution and limited results are presented herein for the sake of completeness. Tables 2 and 3 show the results of minimum compliance optimization of the Lbracket, with filter radii ranging from $r_{\text {min }}=6$ up to $r_{\text {min }}=9$. The projection thresholds are $\eta_{\text {dil }}=0.1$ and $\eta_{\text {ero }}=0.6$ in Table 2 and $\eta_{\text {dil }}=0.4$ and $\eta_{\text {ero }}=0.9$ in Table 3 . The former imposes a larger length scale on the void phase whereas the latter imposes a larger length scale on the solid phase. It can be seen that for the chosen range of filter radii, the same trends of monotonic increase in compliance and decrease in peak stresses are observed. Therefore, it can be concluded that these trends are not a unique property of symmetric projections. Quantitatively, the results show that the symmetric projection provided slightly better trade-offs of compliance and stress, hence the focus from here onwards is on symmetric projections only.

\subsection{The effect of projection thresholds}

It was shown in the previous section that increasing the length scale by increasing the filter radius has a dramatic effect on the trade-off between compliance and stress. Therefore it is interesting to examine whether increasing the length scale by expanding the projection thresholds will have the same outcome. In Table 4 we present several results obtained with the same $r_{\text {min }}=7$ and various symmetric projection thresholds. It can be seen that controlling length scale by projection thresholds does not have a strong effect on the stresses - in fact, increasing the length scale leads to monotonic increase both in compliance and peak stress. The reason for this behavior is the delayed influence of the projection thresholds. Due to the gradual increase in $\beta_{H}$, the increase in length scale is introduced in advanced stages of the optimization when significant (primarily topological) design changes are not possible. Therefore the role of the projection functions in imposing length scale is minor compared to that of the filter radius.

\subsection{Design scenarios without inherent stress concentrations}

The discussion so far was based on the case of an L-bracket, which has an inherent stress concentration due to the definition of the design domain. In the following we present results of minimum compliance optimization of an MBBbeam which does not have such re-entrant corners in the de- sign domain. Therefore, stress concentrations appear only at local regions due to external loads or supports. A symmetric half of the beam is modeled with a grid of $60 \times 180$ finite elements, and a padding layer of 9 elements is added as before. The point load and the point support are distributed over 10 adjacent nodes in order to avoid stress singularities at these regions. Furthermore, solid padding is added again to ensure that the loads and supports are well-connected to the design.

The results in Table 5 show that in such cases, modifying the length scale has only a minor, if any, effect on reducing the peak stresses. The compliance increases monotonically with the length scale as expected, however the maximum stresses decrease only slightly when increasing the filter size from $r_{\text {min }}=3$ to $r_{\text {min }}=5$ and $r_{\text {min }}=7$, and then increase again for $r_{\min }=9$. The length scale does not affect the peak stresses in this example because they appear in the vicinity of the point support and the maximum value is essentially unavoidable. The beam is a statically determinate structure hence the reaction force does not depend upon the design. Once point loads and supports are excluded from the stress criteria, the design domain has no singularities and then the minimum compliance design will also be the one that minimizes stresses - this claim will be further established in the next section. This does not imply that the procedures proposed hereafter are not applicable for cases as the MBB beam that do not have inherent stress singularities. However, for such domains, if a given stress criteria cannot be satisfied by the minimum compliance design after excluding load and support regions, then the whole problem is infeasible and the material volume should be increased.

\section{The relation between compliance and stress}

Minimizing compliance for a structure with fixed Dirichlet boundary conditions, prescribed body forces and tractions is equivalent to reducing the total strain energy. A relation between the strain energy at equilibrium and the stress distribution can be established using the laws of linear elasticity. Based on this relatively simple link, Bendsøe et al (1993) demonstrate that minimization of compliance is equivalent to minimization of an integral of the squared von Mises stress field over the design domain. Such an integral inequality cannot account for the local nature of stress concentrations that appear in singular points - such as re-entrant corners and locations of point loads or supports. Therefore, the stress in such regions cannot be controlled directly by compliance minimization. On the other hand, as discussed in the introduction, the enforcement of strict length scale on both phases removes geometric singularities because it constrains the geometry to consist of a union of disks or balls. Thus, the length scale provides the minimal required smoothness for which there are no stress singularities, while at the same 
Table 1 The effect of filter radius in minimum compliance optimization of an L-bracket, $\eta_{d i l}=0.4, \eta_{\text {ero }}=0.6$.

\begin{tabular}{|c|c|c|c|c|}
\hline Filter radius & 3 & 5 & 7 & 9 \\
\hline Compliance & $2.235 \cdot 10^{2}$ & $2.322 \cdot 10^{2}$ & $2.363 \cdot 10^{2}$ & $2.445 \cdot 10^{2}$ \\
\hline Max. $\sigma_{V M}$ & $6.040 \cdot 10^{-1}$ & $5.449 \cdot 10^{-1}$ & $4.742 \cdot 10^{-1}$ & $4.393 \cdot 10^{-1}$ \\
\hline Volume & $8.983 \cdot 10^{3}$ & $8.968 \cdot 10^{3}$ & $8.964 \cdot 10^{3}$ & $8.961 \cdot 10^{3}$ \\
\hline \multicolumn{5}{|l|}{ Layout } \\
\hline Filter radius & 11 & 13 & 15 & 17 \\
\hline Compliance & $2.578 \cdot 10^{2}$ & $2.764 \cdot 10^{2}$ & $3.052 \cdot 10^{2}$ & $3.319 \cdot 10^{2}$ \\
\hline Max. $\sigma_{V M}$ & $4.303 \cdot 10^{-1}$ & $4.273 \cdot 10^{-1}$ & $4.274 \cdot 10^{-1}$ & $4.408 \cdot 10^{-1}$ \\
\hline Volume & $8.961 \cdot 10^{3}$ & $8.964 \cdot 10^{3}$ & $8.971 \cdot 10^{3}$ & $8.972 \cdot 10^{3}$ \\
\hline Layout & & & & \\
\hline
\end{tabular}

Table 2 The effect of filter radius in minimum compliance optimization of an L-bracket, $\eta_{\text {dil }}=0.1, \eta_{\text {ero }}=0.6$.

\begin{tabular}{lllll}
\hline Filter radius & 6 & 7 & 8 & 9 \\
\hline Compliance & $2.384 \cdot 10^{2}$ & $2.400 \cdot 10^{2}$ & $2.454 \cdot 10^{2}$ & $2.515 \cdot 10^{2}$ \\
Max. $\sigma_{V M}$ & $5.320 \cdot 10^{-1}$ & $4.923 \cdot 10^{-1}$ & $4.778 \cdot 10^{-1}$ & $4.551 \cdot 10^{-1}$ \\
Volume & $8.957 \cdot 10^{3}$ & $8.960 \cdot 10^{3}$ & $8.963 \cdot 10^{3}$ & $8.964 \cdot 10^{3}$ \\
Layout & &
\end{tabular}

time compliance minimization decreases the overall intensity of the von Mises stress field. We note that stress concentrations due to point loads or supports may need to be treated also by adequately distributing the forces - as required in practical design anyway.

The theory for the above discussion in continuous settings is presented in Bendsøe et al (1993). Due to its im- portance for justifying the proposed algorithms we briefly repeat it here. The weak formulation of the linear elasticity problem is given as

$a(\mathbf{u}, \mathbf{v})=l(\mathbf{v}) \quad$ for all $\mathbf{v} \in V_{0}$

where $\mathbf{u}$ is the displacement field, $\mathbf{v}$ is the virtual displacement field and $V_{0}$ is the admissible set of displacement fields. 
Table 3 The effect of filter radius in minimum compliance optimization of an L-bracket, $\eta_{\text {dil }}=0.4, \eta_{\text {ero }}=0.9$.

\begin{tabular}{|c|c|c|c|c|}
\hline Filter radius & 6 & 7 & 8 & 9 \\
\hline Compliance & $2.415 \cdot 10^{2}$ & $2.501 \cdot 10^{2}$ & $2.599 \cdot 10^{2}$ & $2.717 \cdot 10^{2}$ \\
\hline $\operatorname{Max} . \sigma_{V M}$ & $5.103 \cdot 10^{-1}$ & $4.872 \cdot 10^{-1}$ & $4.869 \cdot 10^{-1}$ & $4.810 \cdot 10^{-1}$ \\
\hline Volume & $8.964 \cdot 10^{3}$ & $8.962 \cdot 10^{3}$ & $8.963 \cdot 10^{3}$ & $8.965 \cdot 10^{3}$ \\
\hline Layout & & & & \\
\hline
\end{tabular}

Table 4 The effect of varying symmetric projection thresholds in minimum compliance optimization of an L-bracket, $r_{m i n}=7$.

\begin{tabular}{llll}
\hline Thresholds & $\eta_{\text {dil }}=0.4, \eta_{\text {ero }}=0.6$ & $\eta_{\text {dil }}=0.3, \eta_{\text {ero }}=0.7$ & $\eta_{\text {dil }}=0.2, \eta_{\text {ero }}=0.8$ \\
\hline Compliance & $2.363 \cdot 10^{2}$ & $2.403 \cdot 10^{2}$ & $2.454 \cdot 10^{2}$ \\
Max. $\sigma_{V M}$ & $4.742 \cdot 10^{-1}$ & $4.765 \cdot 10^{-1}$ & $4.879 \cdot 10^{-1}$ \\
Volume & $8.964 \cdot 10^{3}$ & &
\end{tabular}

The bilinear form $a$ and the linear functional $l$ are defined as

$$
\begin{aligned}
a(\mathbf{u}, \mathbf{v}) & =\int_{\Omega}(\mathbf{D}: \varepsilon(\mathbf{u})): \varepsilon(\mathbf{v}) \mathrm{d} \mathbf{x} \\
l(\mathbf{v}) & =\int_{\Omega} \mathbf{f} \cdot \mathbf{v} \mathrm{d} \mathbf{x}+\int_{\Gamma_{N}} \mathbf{t} \cdot \mathbf{v} \mathrm{d} \mathbf{x}
\end{aligned}
$$

with $\varepsilon$ denoting the linearized strain tensor, $\mathbf{D}$ the linear elastic stiffness tensor, $\mathbf{f}$ are body forces and $\mathbf{t}$ are traction forces on the boundary. $\Omega$ is the design domain with boundary $\Gamma=\overline{\Gamma_{D_{i}} \cup \Gamma_{N_{i}}}$ decomposed into two disjoint subsets for each component $u_{i}, i=1,2,3 . \Gamma_{D_{i}}$ is the part of the boundary where $u_{i}=0$ and $\Gamma_{N_{i}}$ denotes the part with prescribed traction $t_{i}$. The goal is to find a solution $\mathbf{u} \in V_{0}$ where

$V_{0}=\left\{\mathbf{v} \in\left[H^{1}(\Omega)\right]^{3}: v_{i}=0\right.$ on $\left.\Gamma_{D_{i}}, i=1,2,3\right\}$.

The compliance functional is computed as

$l(\mathbf{u})=\int_{\Omega}(\mathbf{D}: \varepsilon(\mathbf{u})): \varepsilon(\mathbf{u}) \mathrm{d} \mathbf{x}=\int_{\Omega}(\mathbf{C}: \sigma(\mathbf{u})): \sigma(\mathbf{u}) \mathrm{d} \mathbf{x}$ where u satisfies Equation 7, and in addition the fourthorder stiffness tensor $\mathbf{D}$ by definition is invertable, i.e., the compliance tensor is given by $\mathbf{C}=\mathbf{D}^{-1}$. Given that the von Mises stress $\bar{\sigma}$ is defined at each point by the expression $\bar{\sigma}^{2}=(\mathbf{M}: \sigma(\mathbf{u})): \sigma(\mathbf{u})$ where $\mathbf{M}$ is constant, the following inequality is established (Bendsøe et al 1993)

$\int_{\Omega} \bar{\sigma}^{2} \mathrm{~d} \mathbf{x} \leq C_{\sigma} \int_{\Omega}(\mathbf{C}: \sigma): \sigma \mathrm{d} \mathbf{x}=C_{\sigma} l(\mathbf{u})$.

In other words, the integral of the squared von Mises stresses is bounded by the compliance, while the constant $C_{\sigma}>0$ depends on the specific stiffness tensor and the area or volume of the computational domain. Thus, minimizing the compliance also minimizes the upper bound of the integral of the squared von Mises stress.

When the stress distribution is uniform - i.e. a fully stressed design is optimal - then it is possible to define a compliance target value that corresponds to a desired allowable stress. This is rarely the case (Zhou and Sigmund 2017), however the inequality holds also for non-uniform stress distributions, as long as there is sufficient regularity of the de- 
Table 5 The effect of filter radius in minimum compliance optimization of an MBB-beam, $\eta_{\text {dil }}=0.4, \eta_{\text {ero }}=0.6$.

\begin{tabular}{|c|c|c|}
\hline Filter radius & 3 & 5 \\
\hline Compliance & $1.437 \cdot 10^{2}$ & $1.494 \cdot 10^{2}$ \\
\hline Max. $\sigma_{V M}$ & $8.399 \cdot 10^{-1}$ & $7.921 \cdot 10^{-1}$ \\
\hline Volume & $5.410 \cdot 10^{3}$ & $5.404 \cdot 10^{3}$ \\
\hline Layout & & \\
\hline Filter radius & 7 & 9 \\
\hline Compliance & $1.520 \cdot 10^{2}$ & $1.576 \cdot 10^{2}$ \\
\hline Max. $\sigma_{V M}$ & $7.907 \cdot 10^{-1}$ & $8.262 \cdot 10^{-1}$ \\
\hline Volume & $5.400 \cdot 10^{3}$ & $5.399 \cdot 10^{3}$ \\
\hline Layout & & \\
\hline
\end{tabular}

sign boundary. Because both the void and the solid parts of the design domain, with length scale control, can be considered to be unions of disks in 2-D and balls in 3-D (Wang et al 2011, Lazarov et al 2016), the boundary is always smooth and the stress is finite. It is essential to ensure that the applied loads do not violate the desired upper bound of the von Mises stress. Thus, concentrated forces and supports should be either avoided, or a sufficient region around them should be considered to be a non-design domain (Bendsøe et al 1993). Under these conditions, it will be shown that minimizing compliance can also minimize the maximum stress.

\section{A procedure with adaptive filter radius}

In this section we present a simple algorithmic addition to the standard minimum compliance formulation (5) that enables to limit the peak stresses to a specified value, by controlling the length scale of the design in a global (or uniform) manner. Prior to the formal presentation of the procedure, the motivation for deriving it will be clarified based on a simple demonstrative example.

\subsection{Applying continuation on filter radius}

As shown in the previous section, in the L-bracket case one could observe a reduction in peak stresses when increasing the length scale. Another supporting example is presented herein, where we solve the L-bracket case again, this time with a continuation on the filter radius. All parameters are the same as in the previous section. Every 10th iteration, when $\beta_{H}$ is increased, we also measure the maximum stress on the physical density field $\bar{\rho}^{\text {int }}$ obtained with a sharp projection, $\beta_{H}=16$. This stress is measured on a nearly solidvoid design so issues with quantifying the stress in intermediate density points are circumvented. If the maximum stress exceeds the allowable value, then the filter radius is increased by 1 . This rule is of course a simplification and is rather arbitrary - a more general approach will be presented in the next section. Compared to the results in Table 11, the total number of iterations remains the same (150) however the trade-off between compliance and stress is different.

Two results obtained for different stress limits are presented in Table 6 When increasing the radius gradually from $r_{\text {min }}=5$ to $r_{\text {min }}=9$ and comparing to results in Table 1 we see that the compliance lies between the results obtained with $r_{\text {min }}=7$ and $r_{\text {min }}=9$ while the stress is lower. Similarly, when increasing the radius gradually from $r_{\min }=7$ to $r_{\text {min }}=12$ and comparing to results in Table 1 , we see that 
the compliance resembles the result obtained with $r_{\text {min }}=13$ while the stress is lower. This demonstrates how by controlling the length scale and adapting it according to the stress violations, one can find better trade-offs between compliance and stress, or in other words lower stresses for a given compliance. Therefore it makes sense to derive a procedure that adaptively controls the length scale according to the peak stresses and their desired value.

\subsection{An inner-outer optimization procedure}

Using the simplistic continuation scheme as proposed above, it is not possible to guarantee that the actual stress constraint is satisfied or that the stress level is not too low, i.e. there is a large slack in the constraint. In this section, we present a more general problem formulation where the filter radius is treated as a design variable. The optimization problem consists of two levels or 'loops': an outer one which determines the length scale (parametrized by the filter radius) according to the peak stresses; and an inner one which minimizes compliance for the length scale given by the outer loop. Formally, the outer loop is defined as

$\min _{r_{\min }} \phi_{\text {outer }}=\left|\sigma_{\max }^{V M}\left(\bar{\rho}^{\text {int }}\right)-\sigma_{\max }^{\star}\right|$

where $\sigma_{\max }^{\star}$ is the allowable stress and $\sigma_{\max }^{V M}\left(\bar{\rho}^{i n t}\right)$ is the maximum von Mises stress measured on the intermediate projection of the design $\rho$ that minimizes (5). The overall computational strategy for obtaining stress-constrained designs via a minimum compliance formulation and length scale control is comprised of the following steps:

1. Set allowable stress $\sigma_{\max }^{\star}$.

2. Initialize outer loop: $i=0$, set initial filter radius $r_{i}$.

3. Inner loop: perform a predefined number of iterations towards solving (5) with the current $r_{i}$.

4. Compute the derivative of the maximum stress w.r.t. the filter radius using a forward difference:

$$
\frac{d \sigma_{\max }^{V M}}{d r} \approx \frac{\sigma_{\max }^{V M}\left(r_{i}+\Delta r\right)-\sigma_{\max }^{V M}\left(r_{i}\right)}{\Delta r}
$$

5. Update the filter radius towards solving (13):

$$
r_{i+1}=r_{i}+\operatorname{sgn}\left(\frac{\sigma_{\max }^{\star}-\sigma_{\max }^{V M}\left(r_{i}\right)}{\frac{d \sigma_{\max }^{V M}}{d r}}\right)\left|\frac{\sigma_{\max }^{\star}-\sigma_{\max }^{V M}\left(r_{i}\right)}{\frac{d \sigma_{\max }^{V M}}{d r}}\right|^{0.5}
$$

\section{6. $i=i+1$, repeat steps $3-6$.}

In our numerical experiments, the procedure outlined above worked well with the filter function defined as in Eq. (1) and with the maximum stress $\sigma_{\max }^{V M}\left(r_{i}\right)$ evaluated with the true (non-differentiable) max function. Nevertheless, in principle it is preferable to derive these building blocks with differentiable functions, so that the expression (14) approximates an existing, continuous derivative. The cone-shape filter represented by Eq. (1) is replaced by the Gaussian-shape filter,

$w\left(\mathbf{x}_{j}\right)=\exp \left(-\left|\frac{d\left(\mathbf{x}_{i}, \mathbf{x}_{j}\right)}{\frac{r}{2}}\right|^{2}\right)$

where $d\left(\mathbf{x}_{i}, \mathbf{x}_{j}\right)$ is the distance between the two points $\mathbf{x}_{i}$ and $\mathbf{x}_{j}$, the former representing the center of the filter and the latter representing any point in the domain; and $r$ is the equivalent of the filter radius (Bruns and Tortorelli 2003; Wang and Wang 2005; Sigmund 2007). The Gaussian filter behaves similarly to the standard cone-shape filter but it is continuously differentiable. Furthermore, the maximum von Mises stress $\sigma_{\max }^{V M}\left(r_{i}\right)$ within the domain is approximated using a standard $p$-norm function so that a differentiable evaluation is obtained.

The results of minimum compliance optimization of the L-bracket, using an adaptive filter radius according to the strategy outlined above, are presented in Table 7 For the forward difference computation in Eq. (14), we use a $p$ norm approximation of the maximum stress with an exponent equal to 8 and $\Delta r=r_{i} / 100$. Higher values of $p$ have been used successfully, but we do not find them necessary as the only purpose is to find the directional change in the maximum stress due to a change in length scale. In order to avoid very large design changes when $r_{\text {min }}$ is updated, a move limit of 1.0 is imposed on the difference between $r_{i+1}$ and $r_{i}$. Applying such a move limit is a standard safeguarding technique in many non-linear optimization procedures. In all the examples presented from this point onwards, an inner loop consists of 10 design iterations and the total number of design iterations is 150 . Therefore, the total number of function evaluations is 150 , plus two evaluations for computing the finite difference on the intermediate design, every time the filter is updated. The filter size is updated at the end of an inner loop only if the (true) maximum stress differs from the allowable stress by more than $5 \%$.

The three cases presented in Table 7 differ in the values of the initial filter radius and of the allowable stress. Looking closely at the results, it can be observed that the procedure finds trade-offs of compliance and stress that outperform the naive results of Table 1 . For example, the result in the first column shows a compliance of $2.284 \cdot 10^{2}$ which is in between the compliance values obtained with fixed filter radii of 3 and 5 in Table 1 . At the same time, the maximum stress is $4.957 \cdot 10^{-1}$ which is lower than the stress level of the layout obtained with a fixed radius of 5 - hence the attained trade-off is superior. Furthermore, the procedure is capable of finding the required filter radius so the user does not necessarily need to predict an appropriate value - though choos- 
Table 6 Minimum compliance of an L-bracket with a simple continuation on filter radius, $\eta_{d}=0.4$ and $\eta_{e}=0.6$.

\begin{tabular}{lll}
\hline Initial radius & 5.00 & 7.00 \\
Final radius & 9.00 & 12.00 \\
$\sigma_{\max }^{\star}$ & $4.500 \cdot 10^{-1}$ & $4.100 \cdot 10^{-1}$ \\
Max. $\sigma_{V M}$ & $4.162 \cdot 10^{-1}$ & $3.958 \cdot 10^{-1}$ \\
Compliance & $2.404 \cdot 10^{2}$ & $2.802 \cdot 10^{2}$ \\
Volume & $8.961 \cdot 10^{3}$ &
\end{tabular}

Table 7 Minimum compliance optimization of an L-bracket, with an adaptive filter radius in an outer optimization loop, $\eta_{d}=0.4$ and $\eta_{e}=0.6$.

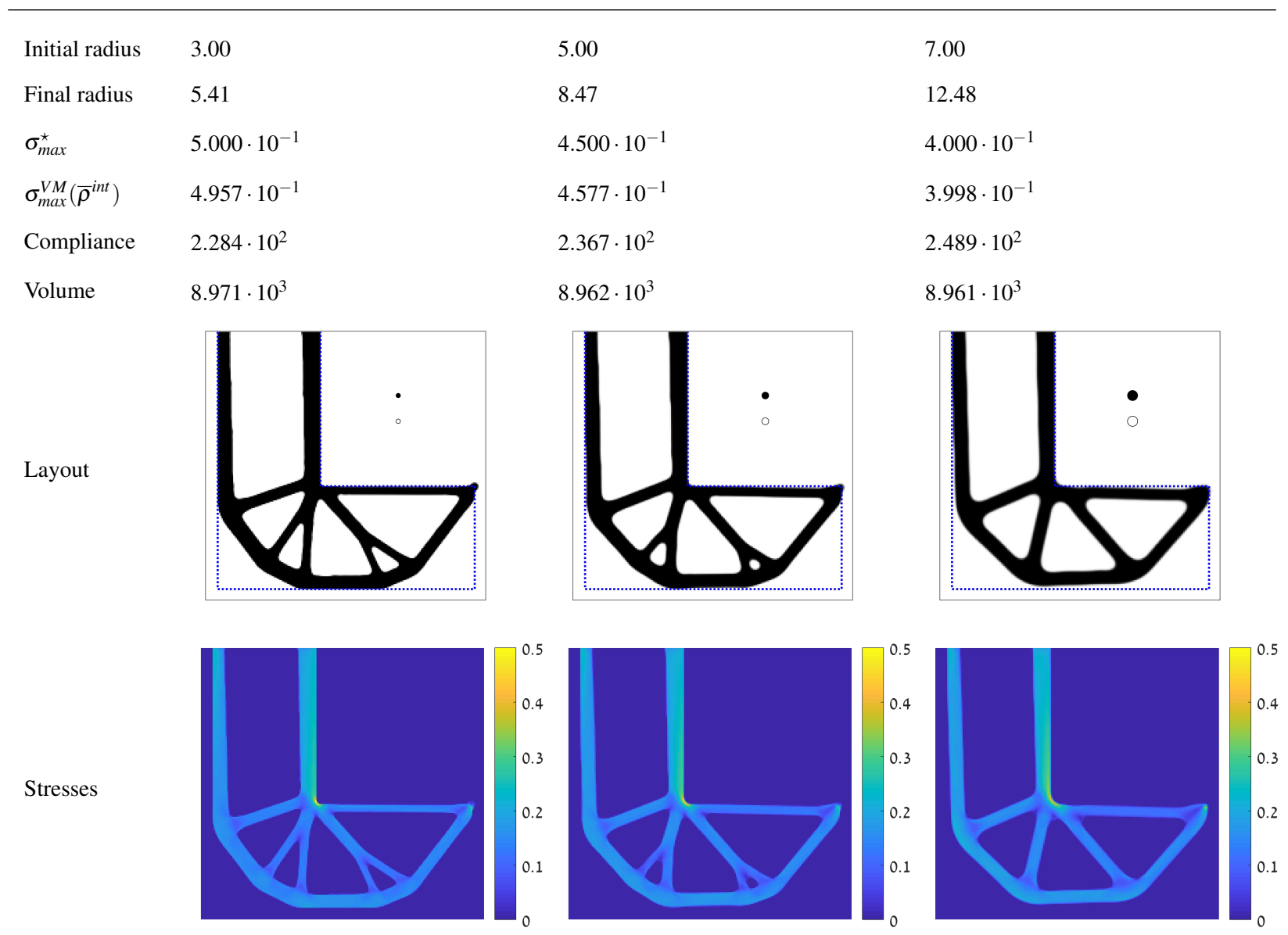



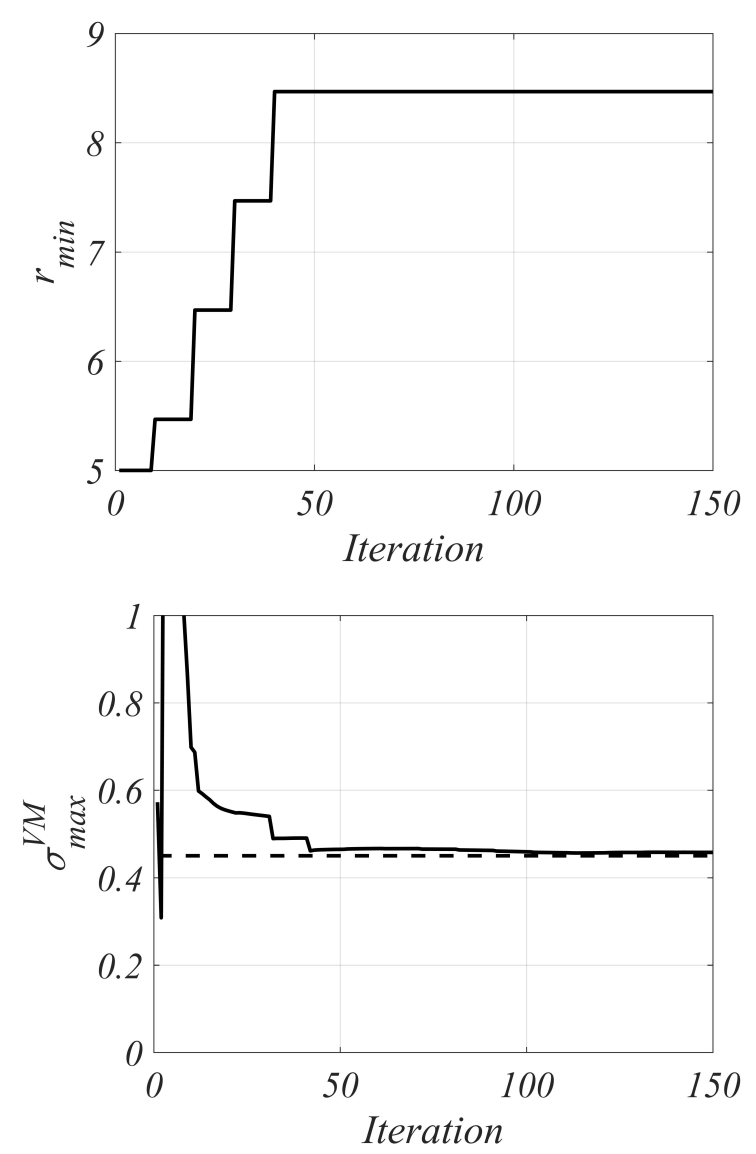

Fig. 2 The evolution of $r_{\min }$ (top) and $\sigma_{\max }^{V M}\left(\bar{\rho}^{i n t}\right)$ (bottom, dashed line shows $\sigma_{\max }^{\star}$ ), in minimum compliance optimization of an L-bracket corresponding to the second column of Table 7

ing a closer initial value will speed up the convergence to the required filter size.

The convergence of $r_{\min }$ and $\sigma_{\max }^{V M}\left(\bar{\rho}^{i n t}\right)$ throughout the 150 design iterations is presented in Figure 2, that corresponds to the central column of Table 7 . There is a clear general trend - the filter radius increases and maximum stress decreases during the optimization process, because the starting point is deliberately chosen such that the filter radius is too small to satisfy the stress requirement. Once the maximum stress approaches the allowable limit up to a specified tolerance, the updates of the filter radius are suppressed (from iteration 50 onwards). A moderate reduction in stresses is still observed over the next 100 iterations, however this is attributed to the overall improvement of the design, in terms of compliance, stress and the volume constraint.

An example with multiple load cases. As the proposed approach relies on an underlying minimum compliance formulation, the extension to multiple loadcases involves the minimization of a weighted compliance. For such cases, the strategy for adapting the filter radius can be extended in two levels. First, the update of the filter radius in steps (4) and
(5) above needs to be determined according to the particular loadcase that represents the worst violation of its corresponding stress constraint. Once the filter radius is updated, it will subsequently affect all loadcases and may reduce the maximum stress also in loadcases that do not have any violation of the stress constraint - hence some conservativeness is introduced. Therefore in the second level, one can manipulate the weights in the weighted compliance objective such that priority is given to loadcases that are more critical w.r.t. maximum stresses. However, according to our experiments this leads to a serious compromise on compliance and does not necessarily contribute to less conservative designs w.r.t. the loadcases with an inactive stress constraint. A more promising approach for reducing conservativeness will be discussed in the next section. The following results are therefore restricted to an update of the filter radius only.

Results with a double L-bracket and two separate loadcases are presented in Figure 3. The load on the left hand side of the structure is 0.5 and the load on the right hand side is 1.0 , while the allowable stress is $\sigma_{\max }^{\star}=4.0 \cdot 10^{-1}$ in the whole domain. As the optimization minimizes a weighted compliance with equal weights ( 0.5 in this case), the result is expected to have more material on the right hand side. But the adaptation of the filter radius is determined according to the derivatives of the maximum stress violation, and then the length scale is altered uniformly so both loadcases will be affected by a certain reduction in maximum stresses. All parameters are the same as for the standard Lbracket, except that the allowable volume fraction is $40 \%$ and the FE grid is increased to $320 \times 200$ so that each side is the same as a standard L-bracket. The initial filter radius is set to $r_{\min }=5.0$ and when keeping it constant, the maximum stresses are $4.638 \cdot 10^{-1}$ and $2.559 \cdot 10^{-1}$ for the loads on the right hand side and left hand side, respectively. The weighted compliance is $2.504 \cdot 10^{2}$. With the given limit of $\sigma_{\text {max }}^{\star}=0.4$, the filter radius is gradually adapted up to the value of $r_{\text {min }}=8.8310$. The maximum stresses are 3.916 . $10^{-1}$ and $2.129 \cdot 10^{-1}$ for the loads on the right hand side and left hand side, respectively. The weighted compliance is naturally higher than before, $2.578 \cdot 10^{2}$. It can be seen that because of the uniform increase in length scale, the slack in the inactive stress constraint (for the load on the left) becomes even larger, as stresses are reduced throughout the domain even if this particular stress constraint is not active. We note that if the loads were chosen to be of the same magnitude and the allowable stresses would be different for each loadcase, while the weights were kept equal, then the weighted minimum compliance would yield a symmetric design - hence some conservativeness with respect to the inactive loadcase will again be introduced. 

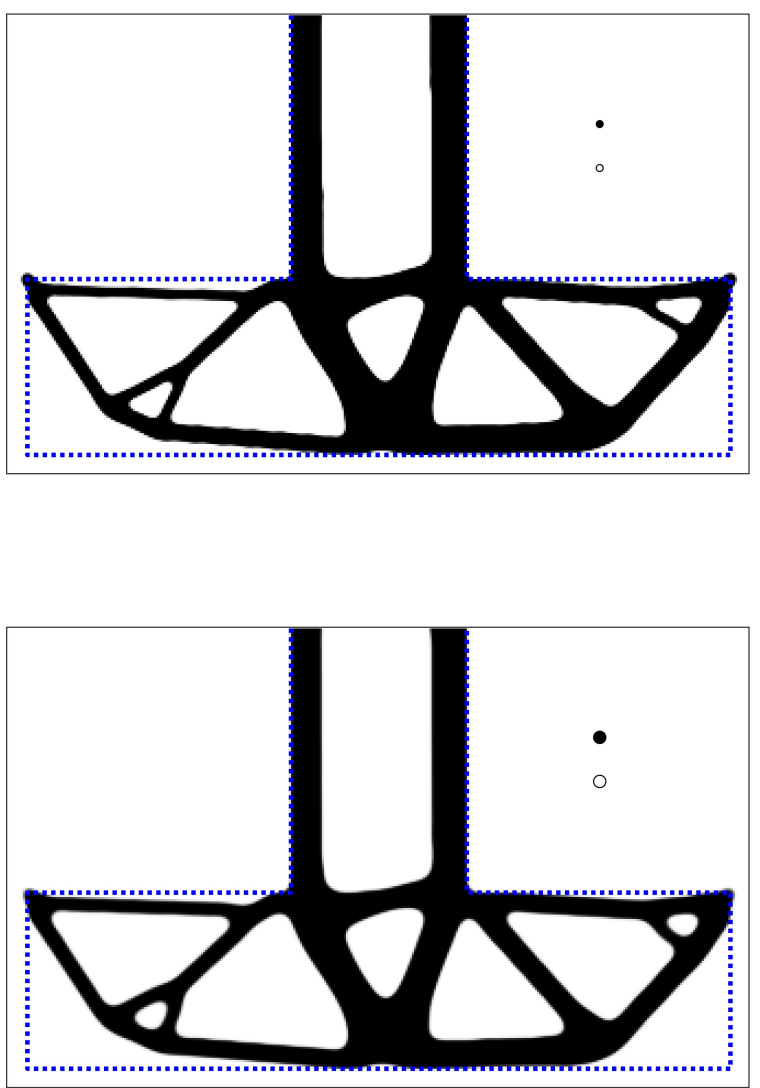

Fig. 3 Minimum weighted compliance optimization of a double Lbracket with two separate loadcases. Top: $r_{\min }=5.0, \sigma_{\max }^{V M}\left(\bar{\rho}^{\text {int }}\right)=$ $4.638 \cdot 10^{-1}$; bottom: adaptive filter radius in an outer optimization loop with $\sigma_{\max }^{\star}=4.0 \cdot 10^{-1}$, initial $r_{\min }=5.0$, final $r_{\min }=8.8310$, $\sigma_{\max }^{V M}\left(\bar{\rho}^{i n t}\right)=3.916 \cdot 10^{-1}$.

\section{A procedure with spatially varying filter radius}

The results so far reveal the significant influence that the length scale has on the stress performance of the optimized design. In the vast majority of cases, a reduction in maximum stress can be achieved by increasing the length scale. However this typically comes with a price - the designs are not as stiff as those that are generated with a small length scale. Looking back at the results in Table 1 , it can be seen that low compliance is achieved when the layout consists of a larger number of thin bars; whereas low stresses are achieved when the layout consists of a small number of thick bars, and more importantly - large radius of curvature at the re-entrant corner. In other words, it is beneficial to have a large length scale near the re-entrant corner so that stresses are reduced, while in the remainder of the domain it is better to have a small length scale that leads to lower compliance. In this section, we propose a procedure that enables to cap- ture the best of both worlds, by using a spatially varying filter radius.

By introducing a spatial variation, the filter radius parameter is treated as a function of the position in space rather than as a constant value. The idea of spatially varying filter radius can and will be combined with the procedure outlined in Section 4 where the filter radius was treated as a design variable that is constant in space. But first we wish to demonstrate the effect of a spatially varying filter using a constant configuration - meaning the filter radius throughout the domain is determined once before the optimization. The distribution of filter radii is based on a basic assumption, that stress concentrations are expected in the vicinity of re-entrant corners and therefore it is necessary to increase the length scale in these regions.

More generally, a re-entrant corner can be seen as a "stress attractor" point. Within a certain influence distance from the stress attractor point, the radius is increased according to a super-Gaussian function. In a 2-D setting, denoting the distance from a certain point $(x, y)$ to the stress attractor point as $d(x, y)$, we define an auxiliary function $\psi(x, y)$ that measures the influence of the attractor as a value between zero and one,

$\psi(x, y)=\exp \left(-\left|\frac{d(x, y)}{D}\right|^{\theta}\right)$

where $D$ is the characteristic influenced distance and $\theta$ determines the sharpness of the function. The spatial filter radius $\hat{r}(x, y)$ is subsequently computed at each point according to the native radius $r_{\min }$ and the auxiliary function,

$\hat{r}(x, y)=(1+\gamma \psi(x, y)) r_{\min }$

where $\gamma$ is a parameter that determines the increase in filter radius at the attractor point. For example, $\gamma=1$ means that the filter radius at the attractor point will be twice the native filter radius, and it will reduce smoothly as the distance from the attractor is increased, according to the function $\psi(x, y)$.

The result of minimum compliance optimization of the L-bracket with a spatially varying filter radius is presented in Figure 4 The native radius is $r_{\min }=5$, the influence distance is $D=50$, the sharpness parameter is $\theta=5$ and the increase in filter size is $\gamma=2$. The compliance of the optimized design is $2.460 \cdot 10^{2}$ and the maximum stress is $3.133 \cdot 10^{-1}$. These values are quite remarkable, as the compliance is similar to that obtained with a constant filter radius of 9 , while the stress is reduced by nearly $30 \%$. It can be seen that the length scale is very large near the re-entrant corner. The solid members are very thick and the radius of curvature at the corner is very large - these two attributes lead to reduced stresses. In the periphery of the design one can observe much thinner features and small holes, complying with the relatively small native filter size. 

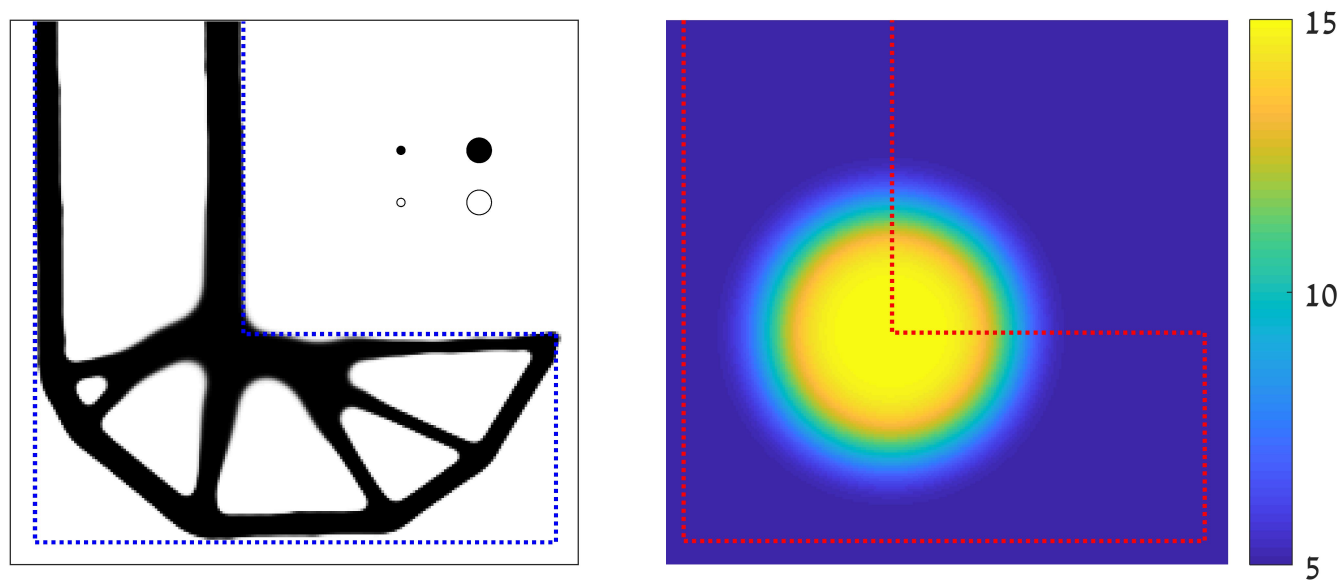

\section{5}

Fig. 4 Minimum compliance optimization of an L-bracket with a spatially varying filter radius. Left: optimized layout; Right: the distribution of filter radii $\hat{r}(x, y)$. The native and increased length scales are plotted as circles alongside the layout.

\subsection{Spatially varying filter radius with inner-outer optimization}

In this section we combine the concept of a spatially varying filter radius, with the adaptive approach presented in Section 4 This can give improved results in comparison to each of the two approaches separately. Three optimization runs of the L-bracket are presented in Table 8 , all have the same initial native filter radius. The input parameters that control the influenced distance from the re-entrant corner and the magnitude of the additional radius are chosen according to the required stress level. Namely, for satisfying a lower allowable stress limit, it is expected that a larger influence distance and a bigger increase in filter radius will be necessary. During the optimization, only the native radius is changed according to the procedure in Section 4. The sharpness of the super-Gaussian function is set to $\theta=2$ in order to obtain a relatively smooth transition. As can be inferred from the results, the combination of spatial variation with adaptivity of the native radius provides superior results compared to each procedure separately. For example, the result in the third column of Table 8 shows lower compliance and lower stress than the result with spatial variation only. The same holds also for the result in the first column of Table 8 when compared to the result in the third column of Table 7 which targeted the same allowable stress.

\subsection{Determining the spatial variation on-the-fly}

The results in this section so far were based on prior knowledge of the problem at hand - the singularity of the design domain of the L-bracket dictates a potential stress attractor point around which the function $\psi$ is defined. In practical cases, it may not be that easy to suggest potential stress attractor points. One possibility is to determine such points based on an initial stiffness optimization without any stress constraints. Another, more general approach is to identify the stress concentrations as the optimization progresses, and to construct a limited number of appropriate $\psi$ functions onthe-fly.

On-the-fly spatial variation is demonstrated using the problem of a U-bracket, that has multiple stress concentrations, see Figure 5 for the setup. The procedure for constructing $\psi$ functions is as follows:

1. Begin minimum compliance optimization with no spatial variation, i.e. a filter radius that is constant in space.

2. If there are violations of the stress constraint:

(a) Find the geometric locations of all points where the stress exceeds the allowable value.

(b) Sort the values by their magnitude for prioritizing the sequence in which $\psi$ functions will be generated.

(c) Remove duplicates - i.e. adjacent points which belong to the same physical stress concentration.

(d) Generate a set of $\psi$ functions that correspond to the attained locations of stress concentrations and update $\hat{r}(x, y)$.

3. Perform a predefined number of iterations towards solving (5) with the current $\hat{r}(x, y)$.

4. Repeat from step 2.

The design domain is based on a $200 \times 100$ grid of square finite elements with side length of 1.0 , from which a square of $50 \times 50$ elements is fixed to be void. The design domain is padded with a layer of 9 void elements on the free boundaries of the design domain. In order to avoid stress concentrations at the loading point, we distribute the load over 9 adjacent nodes at the corner, and pad the design domain locally with 3 layers of solid elements. This ensures that the load is connected to material also after the Heaviside projections. The desired volume fraction of the intermediate design is $V^{\star}=35 \%$ of the U-shape only. As explained above, the optimization begins with 10 design iterations of stan- 
Table 8 Minimum compliance optimization of an L-bracket, with a spatially varying filter radius and adaptivity in an outer optimization loop, $\eta_{d}=0.4$ and $\eta_{e}=0.6$. The native and enlarged filter sizes are plotted alongside the optimized layout.

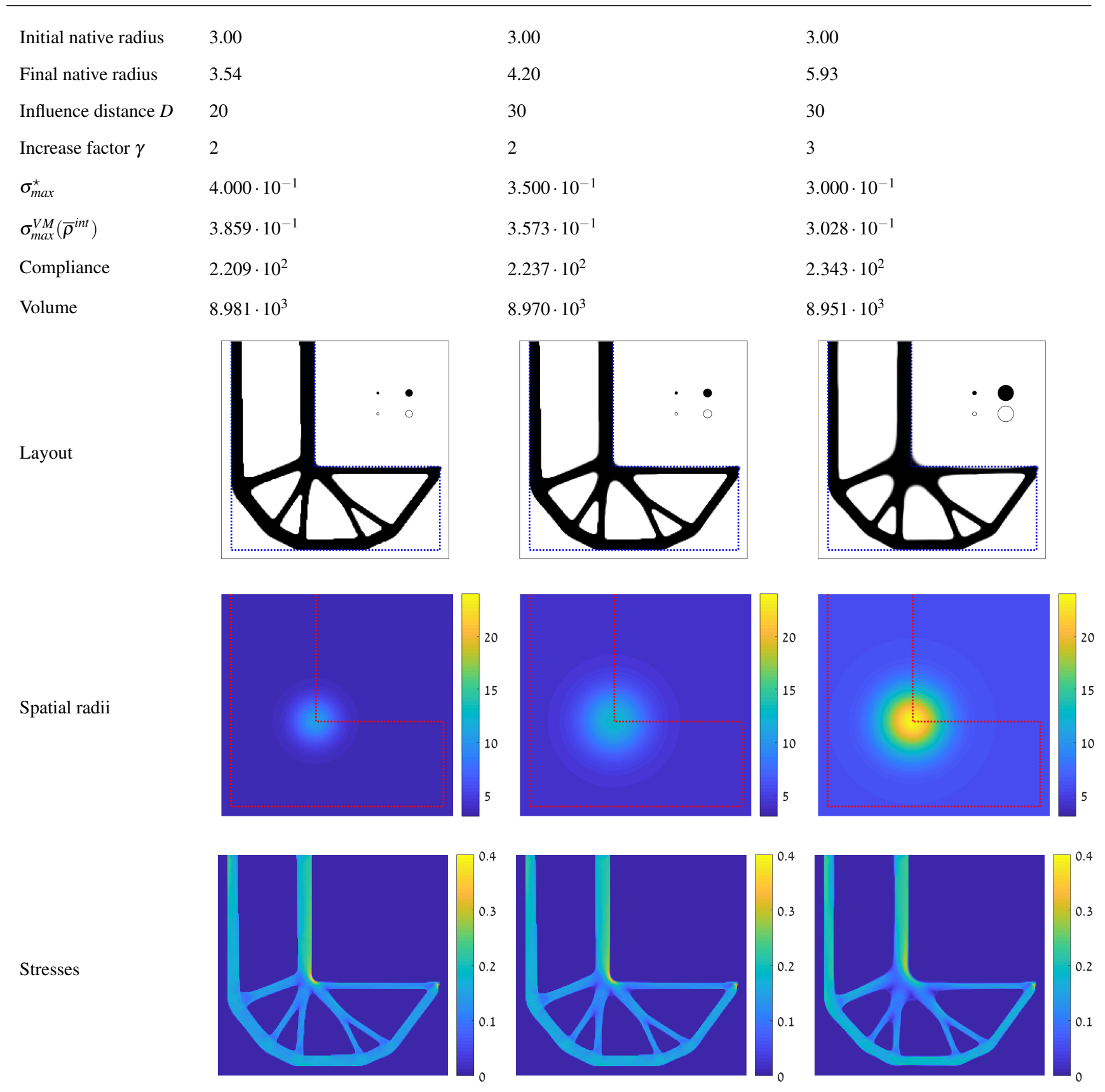

dard minimum compliance with $r_{\text {min }}=3$. Then, every 10 iterations the stresses are checked and sorted for determining the spatial variation $\hat{r}(x, y)$. If there are no stress violations, then the current spatial variation is kept until the next update instance. In implementing step (2c) above, a stress violation point is disregarded if its distance from an existing point (for which a function $\psi$ was already generated, because it has a more acute stress level) is shorter than the influence distance $D$. Finally, the various $\psi$ functions are merged based on the maximum value at each spatial position. In this example, the parameters for generating $\psi$ were set to $\theta=2, \gamma=2$ and $D=20$.

Results with on-the-fly spatial variation, applied to the problem of the U-bracket, are presented in Table 9 . For comparison, we present the results also with standard constant filtering and with an adaptive filter radius which is constant in space, as in Section 4. In the latter case, the radius adapted gradually to $r_{\min }=8.5651$. The quantitative results indicate 


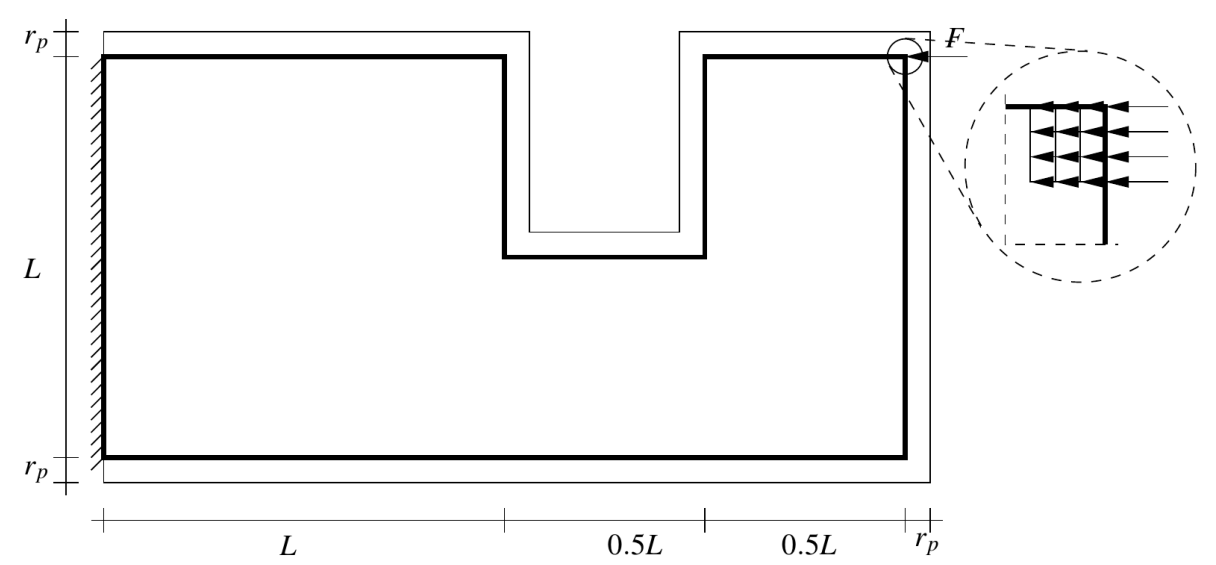

Fig. 5 Design domain and boundary conditions of a U-bracket. For consistent length scale on the boundary of the domain, the $L \times 2 L$ region is padded with a void layer whose thickness is $r_{p}$.

that a stress reduction to the vicinity of the allowable value $\sigma_{\text {max }}^{\star}=0.4$ is possible, either by adapting the filter radius during the optimization or by using a spatial variation based on a mapping of the stress violations. The latter procedure holds the advantage of maintaining high quality in terms of compliance - in fact, it does not yield a higher compliance than the baseline design (note there is a slight difference in the final volume). Looking qualitatively at the optimized layouts, both procedures suggested herein lead to an increased length scale near the re-entrant corners - hence the thicker members and the larger curvature radii. The spatially variable filter yields good stiffness because the length scale away from the re-entrant corners is small, whereas with an adaptive filter radius it is increased uniformly throughout the design domain. This demonstrates the benefit of spatial variation of the length scale, that can be utilized also if there is no prior knowledge regarding the stress concentrations. It is interesting to see that in the U-bracket example, the detection of stress violations was performed only once, after 10 design iterations. Then, based on the generated spatial radii $\hat{r}(x, y)$, the maximum stress decreased to an acceptable level which was specified as within $5 \%$ from the allowable stress. As the optimization progressed, no updates of the spatial variation were necessary because the maximum stress remained within the acceptable range. Therefore in this case the suggested procedure was essentially a standard minimum compliance optimization, with a single step of mapping the stresses and determining the spatial length scale accordingly.

Revisiting an example with multiple load cases. The principle of a spatially varying filter radius is quite useful for accommodating multiple loadcases with different stress requirements. As discussed in Section 4.2, with multiple loadcases the adaptive filter radius procedure can lead to conservative designs w.r.t maximum stresses because the filter is increased uniformly according to the stress violation of the worst loadcase. Using on-the-fly spatial variation, the increase of filter radius can be limited to specific regions of the design that are affected by the loadcases that actually exhibit a stress violation. At the same time, regions with inactive stress constraints will not be affected and therefore their compliance can be better maintained.

We revisit the same example of a double L-bracket as in Section 4.2, this time with two equal loads of magnitude 1.0 but different stress requirements. We use the following parameters: native radius $r_{\min }=5.0 ; \sigma_{\max }^{\star}=0.4$ for the load on the right; $\sigma_{\text {max }}^{\star}=0.6$ for the load on the left; sharpness parameter $\theta=2$; relative radius increase $\gamma=2$; and influence distance $D=20$. The result is presented in Figure 6 . As before, the detection and sorting of stress violations had to be performed only once, after 10 design iterations, yielding the spatial variation displayed in Figure 6. From that point onwards, the optimization ran without any further violations of either stress constraint. It can be seen that the left part of the structure did not require any increase in filter radius because after 10 design cycles the maximum stress was already within the (relatively liberal) allowable limit. In the optimized design, the maximum stresses were $3.110 \cdot 10^{-1}$ for the load on the right and $5.197 \cdot 10^{-1}$ for the load on the left. The compliance values were $2.020 \cdot 10^{2}$ for the load on the right and $2.121 \cdot 10^{2}$ for the load on the left. It is interesting to see how more material was invested in the right side because of the tighter stress requirements, however the compliance due to the load on the left part is only slightly higher - because the smaller length scale compensates for the loss of material. Another interesting aspect is the location of 'stress attractor' points - in this example, besides the expected stress violation in the corner, two additional violations are detected which are on the peripheral bars. Consequently, the length scale in these areas is increased and right side of the design becomes thicker than the left side. 
Table 9 Minimum compliance optimization of a U-bracket, with a standard procedure (left), an adaptive filter radius (middle) and a spatially varying filter radius based on observed stress concentrations (right).

$\begin{array}{llll}\text { Method } & \text { standard } & \text { adaptive } r & \text { spatial } \hat{r} \text { on-the-fly } \\ \sigma_{\max }^{\star} & - & 4.000 \cdot 10^{-1} & 4.000 \cdot 10^{-1} \\ \sigma_{\max }^{V M}\left(\bar{\rho}^{i n t}\right) & 5.057 \cdot 10^{-1} & 4.128 \cdot 10^{-1} & 3.897 \cdot 10^{-1} \\ \text { Compliance } & 1.191 \cdot 10^{2} & 1.275 \cdot 10^{2} & 1.174 \cdot 10^{2} \\ \text { Volume } & 6.150 \cdot 10^{3} & 6.125 \cdot 10^{3} & 6.183 \cdot 10^{3}\end{array}$

Layout
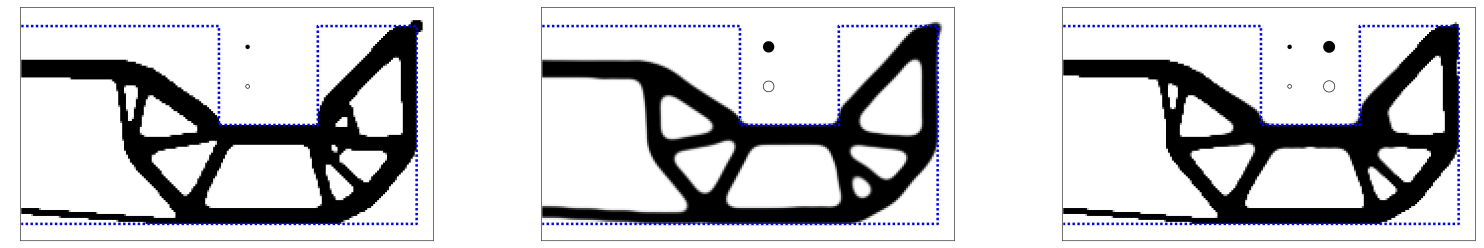

Spatial radii
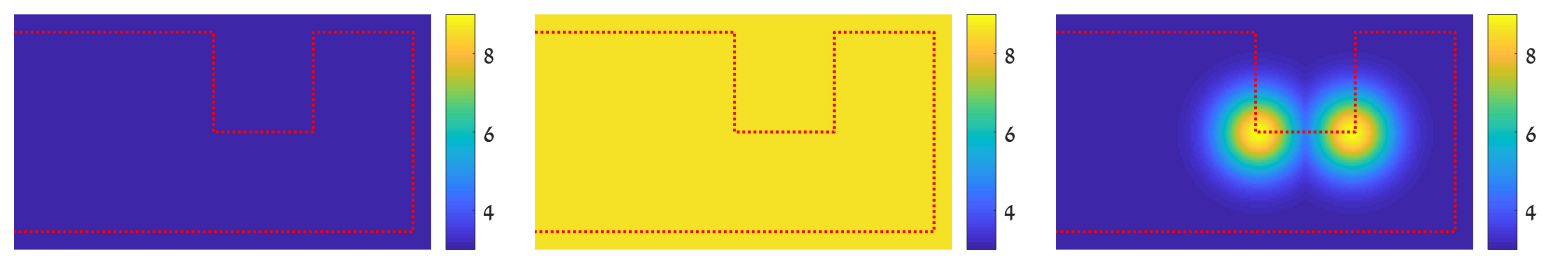

\section{Quantitative comparison to state-of-the-art $p$-norm approach}

This section is dedicated to a one-to-one quantitative comparison versus an established approach to stress-constrained topology optimization. Looking at the literature in the context of density-based procedures, it is clear that the aggregated $p$-norm approach is the most common and therefore we wish to compare the results of the current contribution to an implementation based on a $p$-norm approximation of the maximum stress. It is important to note that to the best of the authors' knowledge, stress-constrained topology optimization with consistent length scale imposition and manufacturability - i.e., convergence to black-and-white designs so no thresholding or post-processing is necessary - was not considered so far. Therefore, the comparison herein is based on our own implementation of the $p$-norm approach with the same length scale and manufacturability requirements as in the results presented in Sections 4 and 5 . In the following, we first describe the formulation and implementation of the $p$-norm approach and later provide a comparison to the results in Table 8 .
6.1 Formulation and implementation of a $p$-norm approach

In formulating the $p$-norm approach, we rely on knowledge and experience that have been accumulated in the research community in recent years, and are reflected in several successful implementations as reviewed in the introduction. Additionally, length scale and manufacturability are guaranteed by utilizing robust topology optimization (Wang et al 2011. Lazarov et al 2016). For minimizing volume subject to stress constraints and a compliance constraint, which reflects the same design intent as the previous formulations in this article, the optimization problem is posed as follows,

$$
\begin{aligned}
\min _{\rho} & \sum_{e=1}^{N_{E}} \bar{\rho}_{e}^{d i l} v_{e} \\
\text { s.t.: } & g_{1}=\mathbf{f}^{T} \mathbf{u}-c_{\text {ero }}^{\star} \leq 0 \\
& g_{2}=\sigma_{P N}-\sigma_{\text {ero }}^{\star} \leq 0 \\
& 0 \leq \rho_{e} \leq 1, \quad e=1, \ldots, N_{E}
\end{aligned}
$$

with: $\mathbf{K}_{e r o} \mathbf{u}=\mathbf{f}$

where $c_{\text {ero }}^{\star}$ is the allowable compliance; $\sigma_{P N}$ is an approximation of the maximum stress; $\sigma_{\text {ero }}^{\star}$ is the allowable stress; 

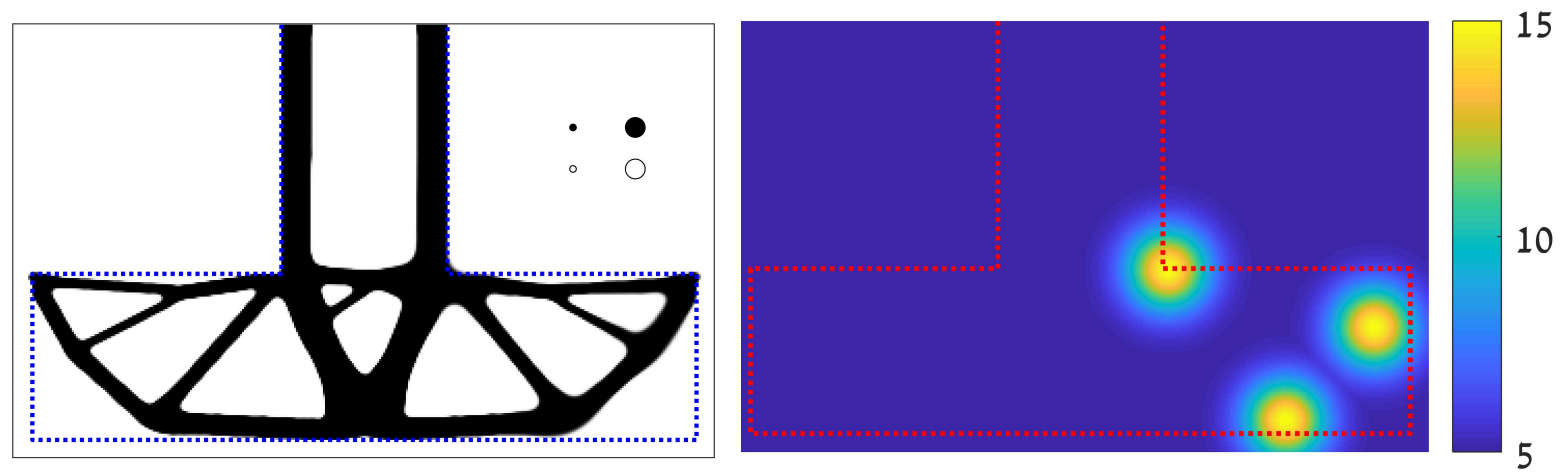

Fig. 6 Minimum weighted compliance optimization of a double L-bracket with two separate loadcases and a spatially varying filter radius. Left: optimized layout; Right: the distribution of filter radii $\hat{r}(x, y)$. The native and increased length scales are plotted as circles alongside the layout.

and all other components were defined in Eq. 57. We note that both constraints $g_{1}$ and $g_{2}$ are imposed on the eroded design which is the only one that is evaluated within every design cycle. Therefore the values $c_{\text {ero }}^{\star}$ and $\sigma_{\text {ero }}^{\star}$ have the subscripts ero and need to be adapted such that eventually the compliance and stress requirements on the intermediate design will be satisfied. Furthermore, $\sigma_{\text {ero }}^{\star}$ needs to be scaled such that eventually the true maximum stress, and not the approximate one, will be lower than the allowable stress.

Based on a series of numerical experiments, the parameters chosen for achieving the best results are:

- SIMP penalization: the stiffness as in Eq. 6) is penalized with $p=2$ whereas the stress at each point is evaluated with $p=0.5$ (e.g. Le et al 2010).

- Smooth Heaviside: because stresses are not evaluated accurately on intermediate densities, the optimization begins with $\beta_{H}=4.0$ which is multiplied by 1.12 every 10 iterations, up to a maximum value of 16 . These settings mimic the progress of $\beta_{H}$ in the results of the previous sections, while considering the higher initial value.

- Constraint aggregation: we use a single $p$-norm approximation with the power of 8 , kept constant throughout the optimization process.

- Constraint values: the values of $c_{\text {ero }}^{\star}$ and $\sigma_{\text {ero }}^{\star}$ are modified every 10 design iterations according to the value of the true constraints. These are $g_{1}^{i n t}=\mathbf{f}^{T} \mathbf{u}^{\text {int }}-c_{\text {int }}^{\star}$ and $g_{2}^{\text {int }}=\sigma_{\text {max }}^{\text {int }}-\sigma_{\text {int }}^{\star}$ which are evaluated on the intermediate design defined by $\beta_{H}=16.0$, while their allowable values are the actual values one wishes to impose on the design. The intermediate design is defined with a sharp Heaviside in order to approach the true response as much as possible.

In all other aspects - e.g. problem setup, boundary conditions, number of iterations - the implementation follows the same assumptions and parameters as described in the previous sections. The only difference is that the problem $[19]$ is solved with MMA (Svanberg 1987) which was not needed in the previous sections. In the authors' opinion, the results obtained with these settings are at least as good as any other published results in the literature that are based on constraint aggregation within a density-based topology optimization framework. Hence we believe that they provide a solid reference for comparison to the proposed procedures that achieve stress constraints only by length scale control.

\subsection{Comparison to Table 8}

Our quantitative comparison focuses on the results from Table 8 because they reveal the full potential of the proposed procedures - having both an adaptive filter radius and the freedom of spatial variation. The compliance values from the table are used as $c_{i n t}^{\star}$ for the $p$-norm optimization and $\sigma_{\text {int }}^{\star}$ is the same as $\sigma_{\text {max }}^{\star}$ in Table 8 . The filter radius is set according to the final native radius in the table so that the minimum length scales match.

Looking at the results in Table 10, it is observed that for all three cases the $p$-norm approach converged successfully and satisfied both the compliance and the stress constraints. In comparison to Table 8, all three layouts have higher volumes. At the same time, the layouts in the second and third column are somewhat stiffer than their respective layouts, and the layout in the second column has also lower stresses these are due to a certain slack in the final values of the constraints. Therefore, one cannot conclude that either approach reaches better trade-offs in terms of compliance, stress and volume.

The proposed procedures that are based on controlling the length scale should not be seen as a direct alternative to traditional approaches. Their underlying formulation is a simple minimum compliance problem while stresses are regulated and reduced by manipulating the length scale of 
Table 10 Minimum volume optimization of an L-bracket with compliance and stress constraints, using a $p$-norm approximation of the maximum stress, $\eta_{d}=0.4$ and $\eta_{e}=0.6$. The filter size is plotted alongside the optimized layout. The values inside the square brackets are the relative difference compared to Table 8

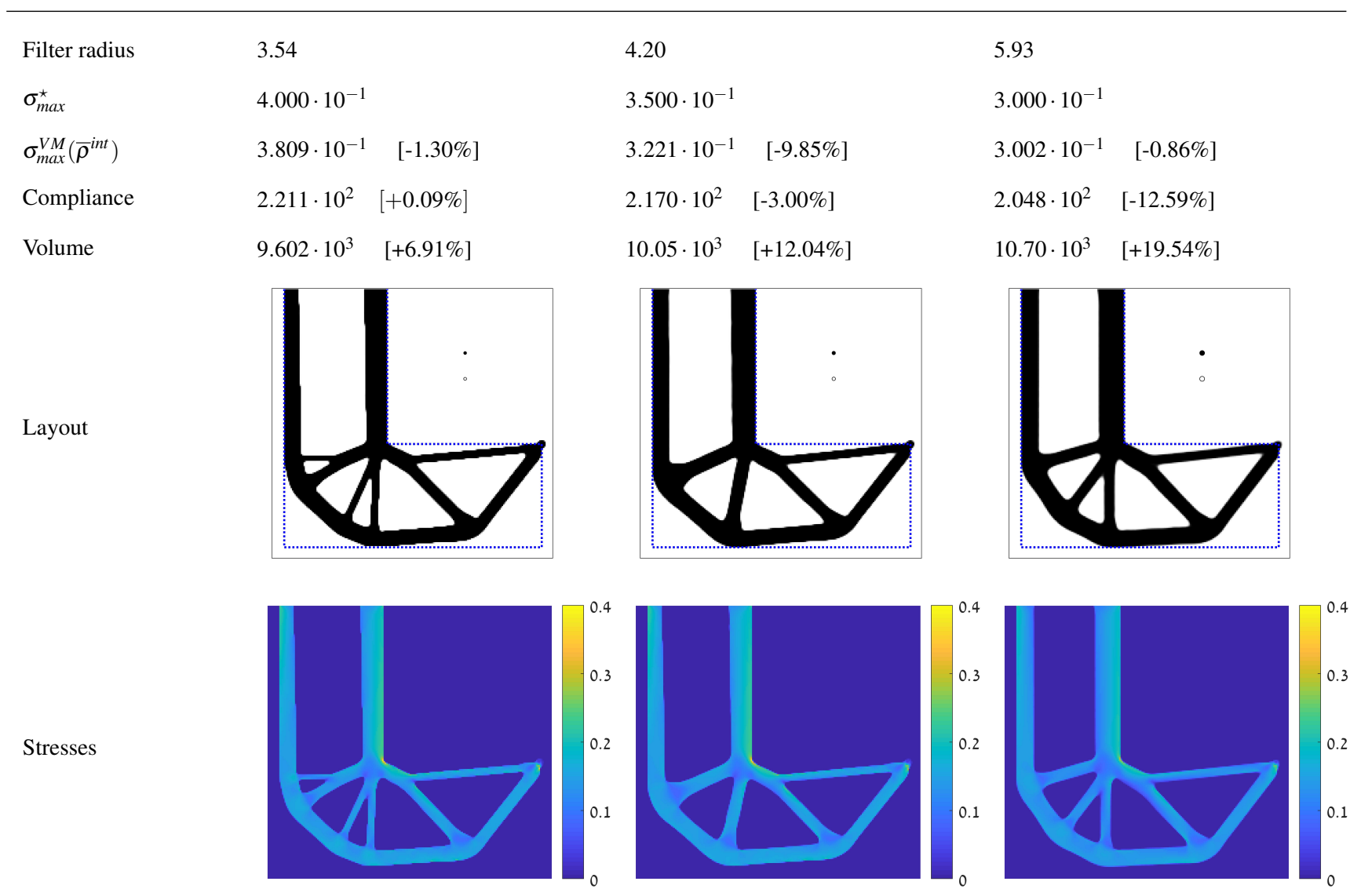

the design. So it is natural that the resulting layouts are different than those obtained with a traditional approach, however the overall trade-off of the three critical quantities is at least as good. From a computational point of view, the proposed procedures do not require computing an adjoint state and can be implemented without a formal optimizer, hence they can serve as an efficient means for finding stiff designs that comply with stress requirements.

\section{Conclusions}

This paper addresses the relation between length scale and maximum stresses in continuum topology optimization. First, the reduction in maximum stress when length scale is increased is demonstrated on classical test cases used for stressbased topology optimization. Then, two different numerical procedures are proposed that essentially offer control over the length scale, according to the allowable maximum stress. Both procedures treat the filter radius, which is the parameter that dominates the attained length scale, as a design variable - in addition to the usual point-wise density variables.
In contrast to typical stress-based procedures that treat the stresses as a function of the topology, we see them as a function of the length scale only. Subsequently, the topology is determined from a standard minimum compliance formulation - which bounds also the stresses according to the inequalities presented by Bendsøe et al (1993).

In the first procedure, the filter radius is adapted throughout the optimization process according to the derivative of the maximum stress w.r.t. the filter radius. In between updates of the filter radius, standard minimum compliance is performed. In the second procedure, a spatially varying filter radius is introduced so that large length scale is obtained where stresses are high, whereas small length scale is maintained elsewhere. It is shown that the optimized designs provide a good trade-off between compliance and stress. From a computational standpoint, the proposed procedures are efficient and simple to implement. The large number of local constraints is avoided, and the well-known difficulties with applying aggregation techniques are circumvented. In essence, the stress-constrained topology optimization is posed as a minimum compliance problem with additional treat- 
ment of the length scale so that sufficient smoothness of the design is guaranteed.

Examining the optimized designs, it can be seen that in some cases the physical density distribution exceeds the prescribed design domain - namely, for enforcing the desired curvature (or void length scale) in re-entrant corners. This is an attribute of the density-based approach where the design domain determines the distribution of the mathematical variables, but it is difficult to constrain the physical densities into the same domain (as discussed also by Clausen and Andreassen (2017)). In cases which require strict enforcement of the design domain on the physical densities, it may be necessary to modify the domain locally so that eventually the physical densities remain within the prescribed boundary. However, in the majority of cases the design domain is merely a simple initial guess for conceptual design, and the designer may not have a clear idea of where large fillets will be necessary. Then, the proposed procedures can be seen as a computational tool for designing the topology and simultaneously determining the required curvatures of re-entrant corners in the design domain.

\section{References}

Allaire G, Jouve F (2008) Minimum stress optimal design with the level set method. Engineering analysis with boundary elements 32(11):909-918

Amir O (2017) Stress-constrained continuum topology optimization: a new approach based on elasto-plasticity. Structural and Multidisciplinary Optimization 55(5):1797-1818

Amstutz S, Novotny AA (2010) Topological optimization of structures subject to von mises stress constraints. Structural and Multidisciplinary Optimization 41(3):407-420

Andreassen E, Clausen A, Schevenels M, Lazarov BS, Sigmund O (2011) Efficient topology optimization in matlab using 88 lines of code. Structural and Multidisciplinary Optimization 43:116, URL http://link.springer.com/article/10.1007\% 2Fs00158-010-0594-7

Bendsøe MP (1989) Optimal shape design as a material distribution problem. Structural optimization 1(4):193-202

Bendsøe MP, Sigmund O (2003) Topology Optimization - Theory, Methods and Applications. Springer, Berlin

Bendsøe MP, Díaz A, Kikuchi N (1993) Topology and generalized layout optimization of elastic structures. In: Bendsøe MP, Soares CAM (eds) Proceedings of the NATO Advanced Research Workshop on Topology Design of Structures, Springer Netherlands, pp 159-205, DOI 10.1007/978-94-011-1804-0_13

Bourdin B (2001) Filters in topology optimization. International Journal for Numerical Methods in Engineering 50:2143-2158

Bruggi M, Duysinx P (2012) Topology optimization for minimum weight with compliance and stress constraints. Structural and Multidisciplinary Optimization 46(3):369-384

Bruggi M, Venini P (2008) A mixed fem approach to stress-constrained topology optimization. International journal for numerical methods in engineering 73(12):1693-1714

Bruns TE, Tortorelli DA (2001) Topology optimization of non-linear elastic structures and compliant mechanisms. Computer Methods in Applied Mechanics and Engineering 190:3443-3459

Bruns TE, Tortorelli DA (2003) An element removal and reintroduction strategy for the topology optimization of structures and com- pliant mechanisms. International journal for numerical methods in engineering 57(10):1413-1430

Clausen A, Andreassen E (2017) On filter boundary conditions in topology optimization. Structural and Multidisciplinary Optimization pp 1-9

De Leon DM, Alexandersen J, Fonseca JS, Sigmund O (2015) Stressconstrained topology optimization for compliant mechanism design. Structural and Multidisciplinary Optimization 52(5):929943

Deaton J, Grandhi R (2014) A survey of structural and multidisciplinary continuum topology optimization: post 2000. Structural and Multidisciplinary Optimization 49(1):1-38, DOI 10.1007/s00158-013-0956-z, URL http://dx.doi.org/10. $1007 / \mathrm{s} 00158-013-0956-z$

Duysinx P, Bendsøe MP (1998) Topology optimization of continuum structures with local stress constraints. International Journal for Numerical Methods in Engineering 43:1453-1478

Duysinx P, Sigmund O (1998) New developments in handling stress constraints in optimal material distribution. In: Proceedings of 7th AIAA/USAF/NASA/ISSMO symposium on multidisciplinary design optimization, AIAA, Saint Louis, Missouri, AIAA Paper, pp 98-4906

Eschenauer HA, Olhoff N (2001) Topology optimization of continuum structures: a review. Applied Mechanics Reviews 54(4):331-389

Fancello E (2006) Topology optimization for minimum mass design considering local failure constraints and contact boundary conditions. Structural and Multidisciplinary Optimization 32(3):229240

Guest JK, Prévost JH, Belytschko T (2004) Achieving minimum length scale in topology optimization using nodal design variables and projection functions. International Journal for Numerical Methods in Engineering 61:238-254

Holmberg E, Torstenfelt B, Klarbring A (2013) Stress constrained topology optimization. Structural and Multidisciplinary Optimization 48(1):33-47

James KA, Waisman H (2014) Failure mitigation in optimal topology design using a coupled nonlinear continuum damage model. Computer Methods in Applied Mechanics and Engineering 268:614631

Kiyono C, Vatanabe S, Silva E, Reddy J (2016) A new multi-p-norm formulation approach for stress-based topology optimization design. Composite Structures 156:10-19

Lazarov BS, Wang F, Sigmund O (2016) Length scale and manufacturability in density-based topology optimization. Archive of Applied Mechanics 86(1-2):189-218

Le C, Norato J, Bruns T, Ha C, Tortorelli D (2010) Stress-based topology optimization for continua. Structural and Multidisciplinary Optimization 41:605-620

Lian H, Christiansen AN, Tortorelli DA, Sigmund O, Aage N (2017) Combined shape and topology optimization for minimization of maximal von mises stress. Structural and Multidisciplinary Optimization 55(5):1541-1557

Madsen S, Lange NP, Giuliani L, Jomaas G, Lazarov BS, Sigmund O (2016) Topology optimization for simplified structural fire safety. Engineering Structures 124:333 - 343

Oest J, Lund E (2017) Topology optimization with finite-life fatigue constraints. Structural and Multidisciplinary Optimization 56(5):1045-1059

París J, Navarrina F, Colominas I, Casteleiro M (2007) Block aggregation of stress constraints in topology optimization of structures. In: Hernández S, Brebbia CA (eds) Computer Aided Optimum Design of Structures X

París J, Navarrina F, Colominas I, Casteleiro M (2010) Block aggregation of stress constraints in topology optimization of structures. Advances in Engineering Software 41(3):433-441 
Park YK (1995) Extensions of optimal layout design using the homogenization method. PhD thesis, University of Michigan, Ann Arbor

Pereira JT, Fancello EA, Barcellos CS (2004) Topology optimization of continuum structures with material failure constraints. Structural and Multidisciplinary Optimization 26(1-2):50-66

Picelli R, Townsend S, Brampton C, Norato J, Kim H (2018) Stressbased shape and topology optimization with the level set method. Computer Methods in Applied Mechanics and Engineering 329:123

Poulsen TA (2003) A new scheme for imposing a minimum length scale in topology optimization. International Journal for Numerical Methods in Engineering 57:741 - 760

Sharma A, Maute K (2017) Stress-based topology optimization using spatial gradient stabilized XFEM. Structural and Multidisciplinary Optimization pp 1-22

Sigmund O (2001) A 99 line topology optimization code written in matlab. Structural and Multidisciplinary Optimization 21:120-127

Sigmund O (2007) Morphology-based black and white filters for topology optimization. Structural and Multidisciplinary Optimization 33:401-424

Sigmund O, Maute K (2013) Topology optimization approaches. Structural and Multidisciplinary Optimization 48(6):1031-1055

Sigmund O, Torquato S (1997) Design of materials with extreme thermal expansion using a three-phase topology optimization method. Journal of the Mechanics and Physics of Solids 45(6):1037-1067

Svanberg K (1987) The method of moving asymptotes - a new method for structural optimization. International Journal for Numerical Methods in Engineering 24:359-373

Thore CJ, Holmberg E, Klarbring A (2017) A general framework for robust topology optimization under load-uncertainty including stress constraints. Computer Methods in Applied Mechanics and Engineering 319:1-18

Verbart A, Langelaar M, van Keulen F (2016) Damage approach: A new method for topology optimization with local stress constraints. Structural and Multidisciplinary Optimization 53(5):1081-1098

Verbart A, Langelaar M, Van Keulen F (2017) A unified aggregation and relaxation approach for stress-constrained topology optimization. Structural and Multidisciplinary Optimization 55(2):663-679

Wang F, Lazarov BS, Sigmund O (2011) On projection methods, convergence and robust formulations in topology optimization. Structural and Multidisciplinary Optimization 43:767-784

Wang MY, Wang S (2005) Bilateral filtering for structural topology optimization. International Journal for Numerical Methods in Engineering 63(13):1911-1938

Xu S, Cai Y, Cheng G (2010) Volume preserving nonlinear density filter based on heaviside functions. Structural and Multidisciplinary Optimization 41(4):495-505, DOI 10.1007/s00158-009-0452-7

Yang R, Chen C (1996) Stress-based topology optimization. Structural Optimization 12(2-3):98-105

Zelickman Y, Amir O (2018) Topology optimization with stress constraints using isotropic damage with strain softening. In: Schumacher A, Vietor T, Fiebig S, Bletzinger KU, Maute K (eds) Advances in Structural and Multidisciplinary Optimization: Proceedings of the 12th World Congress of Structural and Multidisciplinary Optimization (WCSMO12), Springer International Publishing

Zhang S, Gain AL, Norato JA (2017) Stress-based topology optimization with discrete geometric components. Computer Methods in Applied Mechanics and Engineering 325:1-21

Zhou M, Sigmund O (2017) On fully stressed design and p-norm measures in structural optimization. Structural and Multidisciplinary Optimization 56(3):731-736, DOI 10.1007/s00158-017-1731-3 